\title{
The design of solar temperature difference power generation device
}

\author{
Peng Cheng \\ North China Electric Power University, Baoding 071000, China
}

Keywords: Solar energy; temperature difference power generation.

\begin{abstract}
Solar temperature difference power generation technology as a new generation of green environmental protection way, has the characteristics of simple structure, no noise, no pollution, has a broad development prospects. A for solar energy, is developed using semiconductor temperature difference power generation module of solar power systems.
\end{abstract}

\section{Introduction}

Energy is closely related to human survival, it is to improve people's living standard, the material basis for the development of world civilization. The current world Energy consumption is given priority to with fossil resources, including a handful of countries such as China is a coal is given priority to, most of the other countries in oil and gas. According to experts predict that at current consumption, oil, natural gas can only be maintained for less than half a century, most coal can only maintain in one thousand two hundred, the energy crisis is imminent. Therefore vigorously develop renewable energy, with renewable energy and raw material fully replace fossil resources is an important way to solve the energy crisis. Solar energy as a kind of green renewable energy, has the abundant, clean environmental protection, widely distributed, is the ideal alternative energy sources. Temperature difference power generation technology is a kind of solid-state energy conversion technology based on the seebeck effect ${ }^{[1]}$, has features of simple structure, green environmental protection, and high reliability.

\section{The design of solar power systems}

\section{1 focusing principle}

Focusing principle is shown in figure 1.This device to concentrate the sun's rays method is a principle of Fresnel lens concentrator.Using normal convex lens, there will be a corner dim, fuzzy phenomena, this is because only the refraction of light in the medium interface, convex lens piece thicker, the spread of the light in the glass straight line part will make the light attenuation.If you can get rid of the spread of the straight line part, only keep bent surface, can save a lot of material at the same time achieve the same effect of condenser.Fresnel lens is using this principle.Fresnel lens

looks like a piece of countless multiple concentric lines (i.e., Fresnel zone) of the glass, but can reach the effect of the convex lens, if the projection light source is parallel light, together after the projection can maintain the same image brightness everywhere. This device does not need to will have a high temperature, sunshine focused on point, it is designed as the focal plane can form a certain to generate the proper temperature $\left(115 \sim 125{ }^{\circ} \mathrm{C}\right)$, used for heating the hot side of the semiconductor.

\section{2 principle of semiconductor temperature difference power generation}

Principle of semiconductor temperature difference generator is shown in figure 2, the use of p-type and n-type combination of power semiconductor devices. On one side of the device at low temperature, on the other side at high temperature, high temperature device side to side at low temperature and produce heat heat flux, the heat from the hot side into the device, through the device will heat from low temperature side, into the device part of heat energy into electrical energy within the device, by connecting multiple can squeeze more electricity from such components ${ }^{[2-3]}$. 

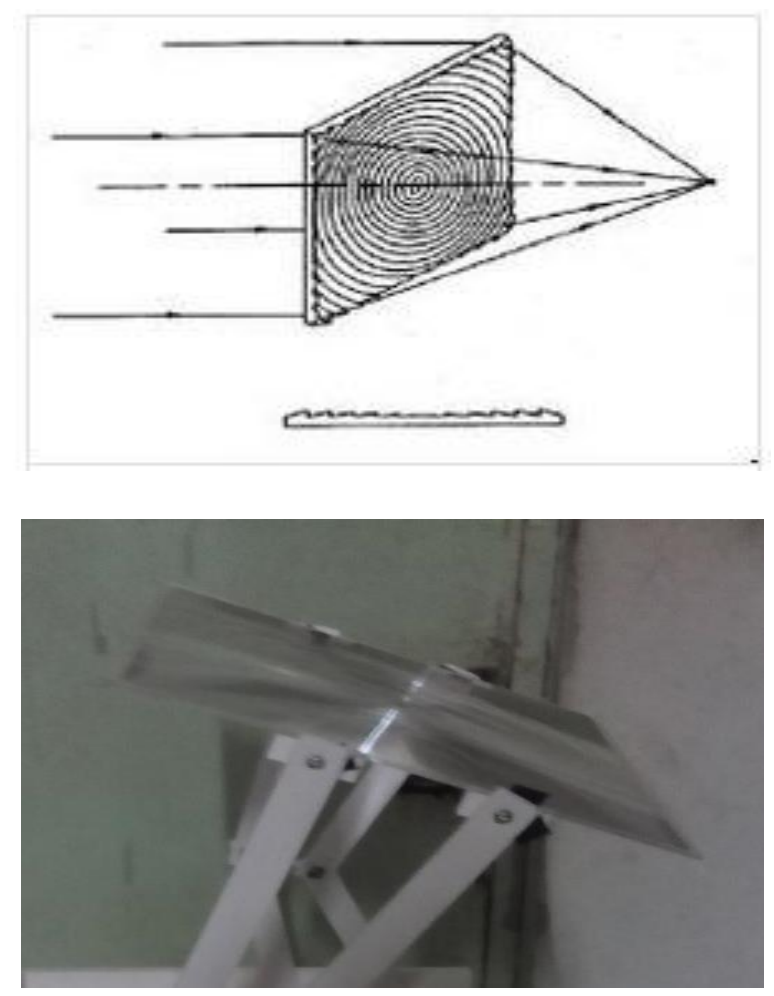

Figure 1 .Fresnel lens

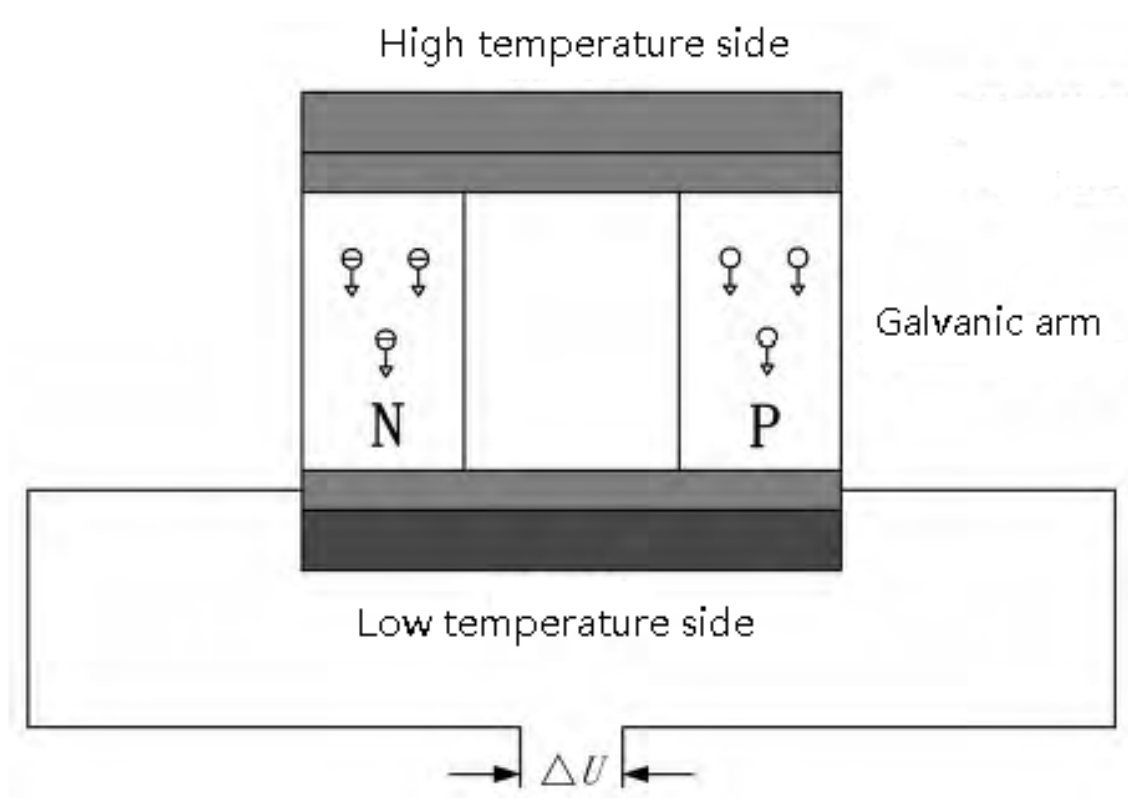

Fig. 2 The principle diagram of the semiconductor temperature difference power generation The model of thermoelectric power generation chip is TEG1-199-1.4-0.5, and the total number of thermoelectric power generation chip is 9 which are arranged between two pieces of graphite sheet in the way of $3 \times 3$. The model is shown in figure 3 and figure 4 .

\subsection{Analysis of The Performance of Thermoelectric Power Generation}

According to the data we get by measuring the different temperature difference between the cold junction and hot junction of thermoelectric power generation chip and the output power under those different conditions, we get the curve of the output power, as Figure 5 shows: 


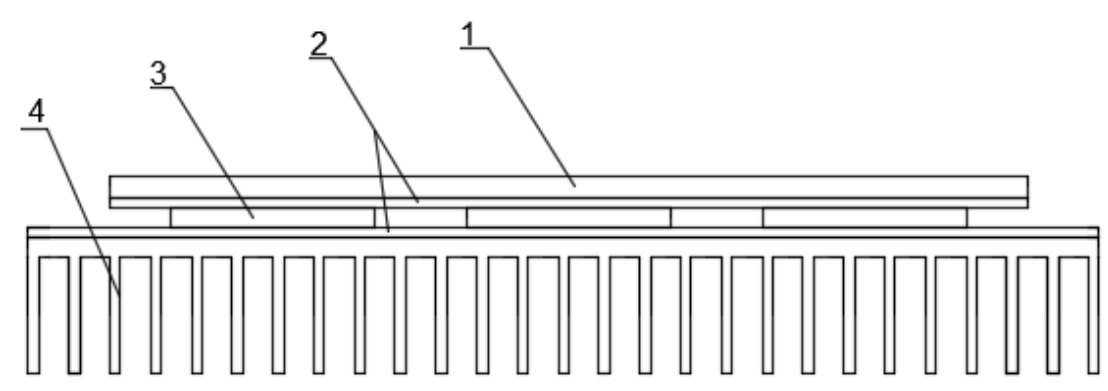

1-Aluminum sheet; 2-Graphite sheet;3-Thermoelectric power generation sheet;4-Air-cooling fin.

Fig. 3 Temperature difference power generation's diagram

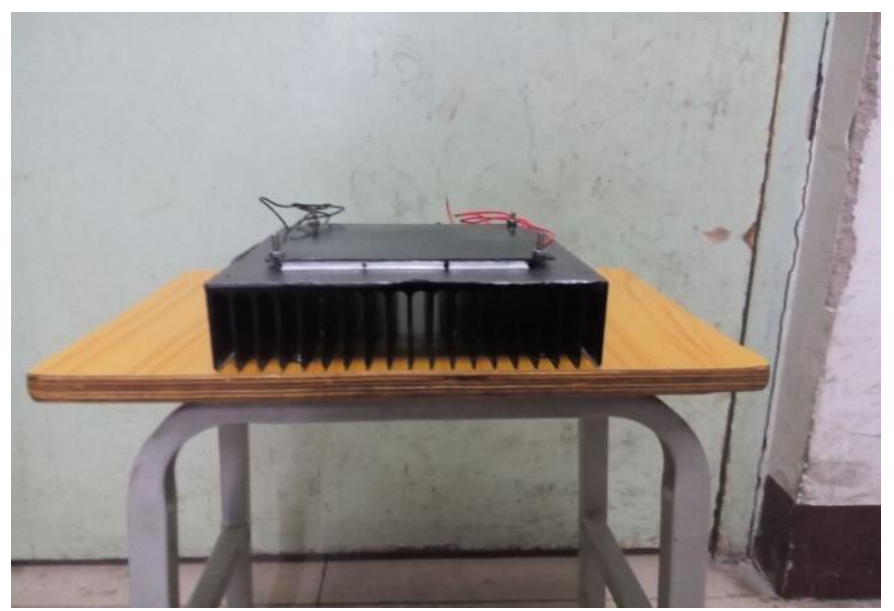

Fig.4 Temperature difference power generation device

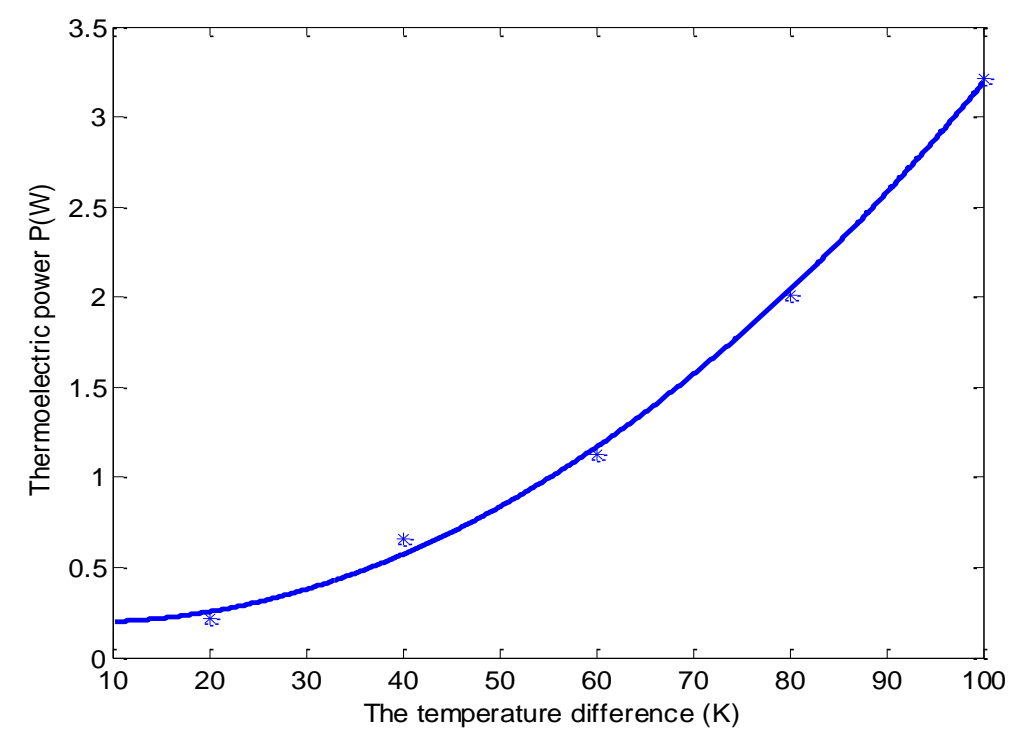

Figure 5. The thermoelectric power with variation of temperature difference

According to the Figure 5, we can draw the conclusion that with the increase of temperature difference between hot and cold junction of the thermoelectric power generation chip, power generation also increase. At the same time, the amount of increase becomes larger. So to increase the output power of the thermoelectric power generation chip, we need to increase the temperature difference between the cold junction and hot junction, which is the key factor to design the device and also the guarantee that the device can work well. 


\section{3. summary}

Energy shortage, resource contention, and such problems as environmental pollution, are threatening the survival and development of human beings.Explore new energy, is the human in the face of the upcoming energy predicament for the sustainable development of an open hand.Solar thermoelectric power generation technology as a kind of green environmental protection way of generating, has wide development prospects.Though the efficiency is low, but as the study of the temperature difference generator optimization and the development of new thermoelectric materials, solar thermoelectric power generation technology will play a more important role in the field of new energy.

\section{References}

[1]Zhao Jianyun,Zhu Dongsheng,Zhou Zeguang,et a1.Research progress of thermoelectric power generation[J].Power Technology,2010,34(3):310-313.

[2] "Yinglin, Huang Hu. Thermal power generation technology and its application[J].Joumal of NalljingNomal University(Engineering and Technology Edition),20 11,11(3):23-30.

[3]Deng Changsheng, the Principle and Application of Solar Energy, Chemical Industry Press, 2010, 35(in Chinese) 